\title{
FABER POLYNOMIALS IN THE THEORY OF UNIVALENT FUNCTIONS
}

\author{
MENAHEM SCHIFFER
}

Introduction. The Faber polynomials play an important role in the theory of univalent functions. Grunsky $[1]^{1}$ succeeded in establishing a set of conditions for a given function which are necessary and in their totality sufficient for the univalency of this function, and in these conditions the coefficients of the Faber polynomials play an important role. Schiffer [2] gave a differential equation for univalent functions solving certain extremum problems with respect to the coefficients of such functions; in this differential equation appears again a polynomial which is just the derivative of a Faber polynomial (cf. Schiffer [3]; see also Schaeffer-Spencer [4]).

It seems, therefore, of interest to study these Faber polynomials more closely, in particular their dependence on the given function with respect to which they are defined, their variation with the latter and certain characteristic inequalities for them and their coefficients. This investigation is carried out in the present paper. In $\$ 1$ we establish a generating function for all Faber polynomials with respect to a given function. In $\$ 2$ we establish variation formulas for the Faber polynomials and their coefficients. In $\$ 3$ we solve certain extremum problems with respect to the coefficients of these polynomials and find again all the inequalities which have been established by Grunsky. In $\$ 4$ we use our method in order to generalize our results and to find inequalities for the Faber polynomials themselves.

1. Identities for Faber polynomials. Consider a function $f(z)$ which has in the neighborhood of $z=\infty$ a development of the form:

$$
f(z)=z+c_{0}+c_{1} z^{-1}+c_{2} z^{-2}+\cdots .
$$

$F_{m}(t)$ is called the $m$ th Faber polynomial with respect to $f(z)$ if it is a polynomial of degree $m$ in $t$ and if we have at $z=\infty$ a development:

$$
F_{m}[f(z)]=z^{m}+\sum_{n=1}^{\infty} c_{m n} z^{-n} .
$$

The existence and uniqueness of all Faber polynomials with respect to a given $f(z)$ are easily shown by recursion.

Presented to the Society, September 5, 1947; received by the editors July 14, 1947.

${ }_{1}$ Numbers in brackets refer to the bibliography at the end of the paper. 
We shall now construct a generating function for all $F_{m}(t)$. For this purpose, we consider the function

$$
U(z, w)=\log \frac{f(z)-f(w)}{z-w}
$$

which is regular for $z$ and $w$ in the neighborhood of infinity. We choose the principal branch of the logarithm, such that if either $z$ or $w$ converge to infinity, $U$ converges to zero, as follows immediately from (1). Hence, we have the following series development for the function $U(z, w)$ of two complex variables:

$$
U(z, w)=\log [f(z)-f(w)]-\log (z-w)=\sum_{m, n=1}^{\infty} d_{m n} z^{-m} w^{-n} .
$$

If we construct next

$$
\log \frac{f(z)-t}{z}=-\sum_{m=1}^{\infty} \frac{1}{m} F_{m}(t) z^{-m},
$$

where the development is valid again in a neighborhood of $z=\infty$ and the $F_{m}(t)$ are polynomials of degree $m$, we find from (5) and (4):

$$
\begin{aligned}
-\sum_{m=1}^{\infty} \frac{1}{m} F_{m}[f(w)] z^{-m} & =\log \frac{f(z)-f(w)}{z-w}+\log \left(1-\frac{w}{z}\right) \\
& =-\sum_{m=1}^{\infty} \frac{1}{m}\left(w^{m}-\sum_{n=1}^{\infty} m d_{m n} w^{-n}\right) z^{-m} .
\end{aligned}
$$

A comparison of equal powers of $z$ on both sides gives finally

$$
F_{m}[f(w)]=w^{m}-\sum_{n=1}^{\infty} m d_{m n} w^{-n}
$$

This shows that the coefficients $F_{m}(t)$ are in fact just the Faber polynomials defined above and that we possess in the left-hand side of (5) a generating function for them.

Comparing (2) and (7), we conclude

$$
c_{m n}=-m d_{m n} \text {. }
$$

Now we have, because of the symmetry of $U(z, w)$ in $z$ and $w$,

$$
d_{m n}=d_{n m}
$$

whence

$$
n c_{m n}=m c_{n m}
$$


an identity previously proved by Grunsky [1] and Schur [5].

Differentiating (5) with respect to $t$, we obtain:

$$
\frac{1}{f(z)-t}=\sum_{m=1}^{\infty} \frac{1}{m} F_{m}^{\prime}(t) z^{-m}
$$

a generating function for the derivatives of Faber's polynomials which will be of use later on.

Numerous further relations might be obtained, as for example the following: Differentiating (11) with respect to $t$ we find:

$$
\frac{1}{(f(z)-t)^{2}}=\sum_{m=1}^{\infty} \frac{1}{m} F_{m}^{\prime \prime}(t) z^{-m}=\sum_{m, n=1}^{\infty} \frac{1}{m n} F_{m}^{\prime}(t) F_{n}^{\prime}(t) z^{-(m+n)}
$$

whence

$$
\frac{1}{k} F_{k}^{\prime \prime}(t)=\sum_{m+n-k} \frac{1}{m n} F_{m}^{\prime}(t) F_{n}^{\prime}(t) .
$$

For our further developments, however, formulas (5), (10) and (11) will be sufficient.

2. A variation formula for the Faber polynomials. Let us suppose now that the function $f(z)$ is regular and univalent in a domain $D$ of the z-plane which contains the point at infinity and is bounded by a finite number of proper continua. It will, therefore, map $D$ conformally upon a domain $\Delta$ of the $\zeta$-plane. If $\zeta_{0}$ is an arbitrary point in the $\zeta$-plane which does not belong to $\Delta$ and $\rho$ any positive constant, there exist infinitely many functions which are univalent in $\Delta$ and have in the domain $\left|\zeta-\zeta_{0}\right|>4 \rho$ a development of the form:

$$
v(\zeta)=\zeta+\frac{a \rho^{2}}{\zeta-\zeta_{0}}+\frac{b \rho^{8}}{\left(\zeta-\zeta_{0}\right)^{2}}+\frac{c \rho^{4}}{\left(\zeta-\zeta_{0}\right)^{3}}+\cdots,
$$

where $|a| \leqq 4^{2},|b| \leqq 4^{3},|c| \leqq 4^{4}, \cdots$ (see Schiffer [2]).

The function

$$
f^{*}(z)=v[f(z)]=f(z)+\frac{a \rho^{2}}{f(z)-\zeta_{0}}+\cdots
$$

is again regular and univalent in the initial domain $D$ and has still a development of the form (1) at infinity. It maps the domain $D$ upon a new domain $\Delta^{*}$ in the $\zeta$-plane. If $\rho$ is small enough, $\Delta^{*}$ will be very near to $\Delta$ and we may conceive the mapping (14) as a small variation of this domain. Variations of the type (15) which transform a univalent function $f(z)$ into a function $f^{*}(z)$ of the same type are of great 
value in solving extremum problems within the class of normalized univalent functions.

Suppose now that to a given univalent function $f(z)$ the $n$th Faber polynomial $F_{n}(t)$ has been determined. If we subject $f(z)$ to a variation (15), how does the corresponding Faber polynomial change? In order to settle this question, we make use of the generating function (5). We have in view of (15)

$$
\begin{aligned}
-\sum_{m=1}^{\infty} \frac{1}{m} F_{m}^{*}(t) z^{-m} & =\log \frac{f^{*}(z)-t}{z} \\
& =\log \frac{f(z)-t}{z}+\frac{a \rho^{2}}{[f(z)-t]\left[f(z)-\zeta_{0}\right]}+o\left(\rho^{2}\right),
\end{aligned}
$$

where $o\left(\rho^{2}\right)$ shall denote henceforth a term containing at least $\rho^{3}$ as a factor. We transform (16) by making again use of identity (5) and applying (11):

$$
\begin{aligned}
-\sum_{m=1}^{\infty} \frac{1}{m} F_{m}^{*}(t) z^{-m}= & -\sum_{m=1}^{\infty} \frac{1}{m} F_{m}(t) z^{-m} \\
& +a \rho^{2} \sum_{m=1}^{\infty} \frac{1}{m} \frac{F_{m}^{\prime}(t)-F_{m}^{\prime}\left(\zeta_{0}\right)}{t-\zeta_{0}} z^{-m}+o\left(\rho^{2}\right) .
\end{aligned}
$$

Comparison of equal powers of $z$ on both sides gives, therefore, the following variation formula for $F_{m}(t)$ :

$$
F_{m}^{*}(t)=F_{m}(t)-a \rho^{2} \frac{F_{m}^{\prime}(t)-F_{m}^{\prime}\left(\zeta_{0}\right)}{t-\zeta_{0}}+o\left(\rho^{2}\right) .
$$

Let us now consider the value $F_{m}[f(w)]$ as a functional of $f(z)$ for a fixed point $w \in D$. Its variation is given by:

$$
F_{m}^{*}\left[f^{*}(w)\right]=F_{m}^{*}[f(w)]+a \rho^{2} \frac{F_{m}^{\prime}[f(w)]}{f(w)-\zeta_{0}}+o\left(\rho^{2}\right),
$$

whence, in view of (18),

$$
F_{m}^{*}\left[f^{*}(w)\right]=F_{m}[f(w)]+a \rho^{2} \frac{F_{m}^{\prime}\left(\zeta_{0}\right)}{f(w)-\zeta_{0}}+o\left(\rho^{2}\right) .
$$

Using the series development (2) for $F_{m}[f(w)]$, an analogous representation for $F_{m}^{*}\left[f^{*}(w)\right]$, and the identity (11) for the second righthand term, we obtain by comparison of the coefficients of $z^{-n}$ on both sides: 


$$
c_{m n}^{*}=c_{m n}+a \rho^{2} \cdot \frac{1}{n} F_{m}^{\prime}\left(\zeta_{0}\right) F_{n}^{\prime}\left(\zeta_{0}\right)+o\left(\rho^{2}\right)
$$

This variation formula for the $c_{m n}$ exhibits, of course, again the symmetry of the matric $n c_{m n}$.

Finally, let us differentiate (15) with respect to $z$; we obtain:

$$
f^{\prime *}(z)=f^{\prime}(z)\left[1-\frac{a \rho^{2}}{\left(f(z)-\zeta_{0}\right)^{2}}\right]+o\left(\rho^{2}\right),
$$

which may be written as a variation formula for $\log f^{\prime}(z)$ as follows:

$$
\log f^{\prime *}(z)=\log f^{\prime}(z)-\frac{a \rho^{2}}{\left(f(z)-\zeta_{0}\right)^{2}}+o\left(\rho^{2}\right),
$$

and which will be used in this form later on. By differentiating (20) with respect to $w$ and applying (22) we get after elementary transformations:

$$
F_{m}^{\prime *}\left[f^{*}(w)\right]=F_{m}^{\prime}[f(w)]+a \rho^{2} \frac{F_{m}^{\prime}[f(w)]-F_{m}^{\prime}\left(\zeta_{0}\right)}{\left(f(w)-\zeta_{0}\right)^{2}}+o\left(\rho^{2}\right) .
$$

This is a variation formula for the functional $F_{m}^{\prime}[f(w)]$ and is interesting in that it contains only the functional itself in addition to $f(w)$ and $\zeta_{0}$.

3. Application of the variation formulas in extremum problems. We use now the formula (21) in order to solve certain extremum problems with respect to the family $\Phi$ of all functions (1) which are univalent in $D$. Let $x \equiv\left(x_{1}, x_{2}, \cdots, x_{N}\right)$ denote a vector of $N$ complex numbers, not all zero, and consider the quadratic form

$$
Q(x, x)=\sum_{m, n=1}^{N} n c_{m n} x_{m} x_{n}
$$

For each given function $f(z)$ of the family $\Phi$ this form has a well defined complex value. We ask now the following question:

What is the maximum modulus of $Q(x, x)$, if $f(z)$ is a function of the family $\Phi$, and for which functions $f(z) \in \Phi$ is this maximum attained?

It follows easily from the theory of normal families that this question is significant and that there exists at least one $f(z) \in \Phi$ for which the maximum value is obtained. We may, therefore, restrict ourselves to the task of characterizing these extremal functions and computing the corresponding value of $|Q(x, x)|$. 
Suppose that we know already an extremal function $f(z)$ and the corresponding value of $Q(x, x) \cdot f(z)$ will map $D$ conformally upon a domain $\Delta$ in the $\zeta$-plane. Let $\zeta_{0}$ be a point in this plane which does not belong to $\Delta$ and subject $\Delta$ to a variation (14) as described in \$2. Under this variation $\Delta$ is transformed into a domain $\Delta^{*}$ which is obtained from $D$ by means of a mapping with a function $f^{*}(z)$, given by (15). Since $f^{*}(z)$ also belongs to the family $\Phi$, its corresponding value $\left|Q^{*}(x, x)\right|$ cannot be larger than the maximal $|Q(x, x)|$. On the other hand, we may easily compute $\left|Q^{*}(x, x)\right|$ by means of formula (21). We have, in view of this formula,

$$
Q^{*}(x, x)=Q(x, x)+a \rho^{2}\left(\sum_{n=1}^{N} x_{n} F_{n}^{\prime}\left(\zeta_{0}\right)\right)^{2}+o\left(\rho^{2}\right) .
$$

The extremal property of $|Q(x, x)|$ implies

$$
\begin{aligned}
\left|Q^{*}(x, x)\right| & =\left|Q(x, x)+a \rho^{2}\left(\sum_{n=1}^{N} x_{n} F_{n}^{\prime}\left(\zeta_{0}\right)\right)^{2}+o\left(\rho^{2}\right)\right| \\
& \leqq|Q(x, x)|,
\end{aligned}
$$

which is equivalent to

$$
\operatorname{Re}\left\{a \rho^{2} Q^{-1}(x, x)\left(\sum_{n=1}^{N} x_{n} F_{n}^{\prime}\left(\zeta_{0}\right)\right)^{2}+o\left(\rho^{2}\right)\right\} \leqq 0 .
$$

This inequality has to be fulfilled for every function $v(\zeta)$ as defined in (14). We now make use of the following lemma (Schiffer [2]):

Let $\Delta$ be a domain in the $\zeta$-plane whose complement consists of continua $\Gamma_{\nu}(\nu=1, \cdots, c)$ and $\sigma(\zeta) \not \equiv 0$ a function analytic in each of the $\Gamma_{\nu}$. Let, further, the inequality

$$
\operatorname{Re}\left\{a \rho^{2} \sigma\left(\zeta_{0}\right)\right\}+o\left(\rho^{2}\right) \leqq 0
$$

hold for every function (14), univalent in $\Delta$. Then each $\Gamma_{\nu}$ is an analytic curve with the parametric representation $\zeta(s)$ and satisfying for properly chosen (real) parameter $s$ the differential equation

$$
\zeta^{\prime}(s)^{2} \sigma\{\zeta(s)\}=1 .
$$

Application of this lemma to the particular inequality (28) leads to the result: The extremal function $f(z)$ maps the domain $D$ upon a domain $\Delta$ in the $\zeta$-plane which is bounded by analytic slits $\zeta(s)$ each of which satisfies the differential equation

$$
\zeta^{\prime}(s)^{2} Q(x, x)^{-1}\left(\sum_{m=1}^{N} x_{m} F_{m}^{\prime}[\zeta(s)]\right)^{2}=1 .
$$


We see now that our particular extremum problem leads to a differential equation (30) with a term $\sigma(\zeta)$ which is a complete square. Thus, this differential equation becomes immediately integrable in closed form. Among all extremum problems arising in the theory of univalent functions, the particular class of those problems with the above property plays an important role, since these problems permit a particularly easy solution.

We conclude from (31) that the boundary curves $\Gamma$, of $\Delta$ satisfy the requirement

$$
\begin{aligned}
& \operatorname{Im}\left\{Q(x, x)^{-1 / 2} \sum_{m=1}^{N} x_{m} F_{m}(\zeta)\right\}=k_{\nu}, \\
& \quad \zeta \in \Gamma_{\nu} ; k_{\nu}=\text { constant } ; \nu=1,2, \cdots, c .
\end{aligned}
$$

The function

$$
S(z)=Q(x, x)^{-1 / 2} \sum_{m=1}^{N} x_{m} F_{m}[f(z)]
$$

is regular in $D$, except for an $N$ th order pole at infinity and possesses constant imaginary parts on each boundary continuum of $D$.

In order to exploit these conditions, we introduce the following class of functions with respect to $D$. We define associate pairs of functions $A_{m}(z)$ and $B_{m}(z)$, having at $z=\infty$ the respective developments

$$
\begin{aligned}
& A_{m}(z)=z^{m}+\sum_{n=1}^{\infty} a_{m n} z^{-n}, \\
& B_{m}(z)=\sum_{n=1}^{\infty} b_{m n} z^{-n}
\end{aligned}
$$

which are regular in $D$ except for the pole of $A_{m}(z)$ at infinity. On each boundary continuum of $D$ we require:

$$
A_{m}(z)=\overline{B_{m}(z)}+\text { const. }
$$

where the complex constant depends on $m$ and the particular boundary continuum. The existence and uniqueness of these functionpairs is easily proved (cf. Grunsky [1]).

We construct now the function

$$
T(z)=Q(x, x)^{-1 / 2} \sum_{m=1}^{N} x_{m} A_{m}(z)+\overline{Q(x, x)^{-1 / 2}} \sum_{m=1}^{N} x_{m} B_{m}(z) .
$$


This function has at $z=\infty$ the same principal part as $S(z)$ and, in view of the characteristic property (34) of $A_{m}(z)$ and $B_{m}(z)$, it has on each boundary continuum of $D$ a constant imaginary part. The function $S(z)-T(z)$ is, therefore, regular throughout $D$ and has on each boundary continuum a constant imaginary part. Hence, it follows by the usual considerations that this function is a constant, that is,

$$
\begin{aligned}
Q(x, x)^{-1 / 2} \sum_{m=1}^{N} x_{m} F_{m}[f(z)]= & Q(x, x)^{-1 / 2} \sum_{m=1}^{N} x_{m} A_{m}(z) \\
& +\overline{Q(x, x)^{-1 / 2}} \sum_{m=1}^{N} \bar{x}_{m} B_{m}(z)+C .
\end{aligned}
$$

Comparing in this equation the terms in $z^{0}$, we find from (2), (33), and $\left(33^{\prime}\right)$ at once that $C=0$. Let us introduce the real parameter $\delta$ by the definition

$$
e^{i \delta}=\operatorname{sgn} Q(x ; x) ;
$$

then (36) may be written in the form

$$
\sum_{m=1}^{N} x_{m} F_{m}[f(z)]=\sum_{m=1}^{N} x_{m} A_{m}(z)+e^{i \delta} \sum_{m=1}^{N} \bar{x}_{m} B_{m}(z) .
$$

Let us now apply the formulas (2), (33), and (33'); comparing the coefficients of $z^{-n}$ on both sides yields:

$$
\sum_{m=1}^{N} x_{m} c_{m n}=\sum_{m=1}^{N}\left(x_{m} a_{m n}+e^{i \delta} \bar{x}_{m} b_{m n}\right) \text {. }
$$

Multiply the $n$th equation with $n x_{n}$ and add all resulting equations for $n=1$ till $n=N$. We obtain:

$$
\sum_{m, n=1}^{N} n c_{m n} x_{m} x_{n}=\sum_{m, n=1}^{N} n a_{m n} x_{m} x_{n}+e^{i \delta} \sum_{m, n=1}^{N} n b_{m n} \bar{x}_{m} x_{n},
$$

whence:

$$
\left|\sum_{m, n=1}^{N} n c_{m n} x_{m} x_{n}\right| \leqq\left|\sum_{m, n=1}^{N} n a_{m n} x_{m} x_{n}\right|+\sum_{m, n=1}^{N} n b_{m n} \bar{x}_{m} x_{n} .
$$

The last right-hand side term is non-negative, since it can be shown that $\left(n b_{m n}\right)$ is a positive-definite Hermitian matric; we mention here that $\left(n a_{m n}\right)$ is a symmetric matric (cf. Grunsky [1]).

The significant feature of $\left(39^{\prime}\right)$ is that the right-hand terms depend only upon $D$ and not upon the mapping function $f(z)$. The in- 
equality (39'), proved for the extremal function, holds, therefore, a fortiori for every other admissible function also.

The form of (39) leads us to the following extremum problem:

What is the maximum modulus of $P(x, x)=Q(x, x)-\sum_{m, n=1}^{N} n a_{m n} x_{m} x_{n}$, if $f(z)$ is a function of the family $\Phi$ ?

One sees easily that the above considerations have to be repeated and that the same sequence of formulas appears, except that instead of $Q(x, x)$ we have always to introduce $P(x, x)$. We obtain finally, by putting

$$
e^{i \gamma}=\operatorname{sgn} P(x, x),
$$

the equation

$$
\sum_{m, n=1}^{N} n\left(c_{m n}-a_{m n}\right) x_{m} x_{n}=e^{i \gamma} \sum_{m, n=1}^{N} n b_{m n} \bar{x}_{m} x_{n}
$$

for the coefficients $c_{m n}$ belonging to the extremal function $f(z)$. Hence, we have in general:

$$
\left|\sum_{m, n=1}^{N} n\left(c_{m n}-a_{m n}\right) x_{m} x_{n}\right| \leqq \sum_{m, n=1}^{N} n b_{m n} \bar{x}_{m} x_{n} .
$$

These are just Grunsky's inequalities, derived here by variational methods. We have obtained, moreover, the following equation for extremal functions:

$$
\sum_{m=1}^{N} x_{m} F_{m}[f(z)]=\sum_{m=1}^{N} x_{m} A_{m}(z)+e^{i \gamma} \sum_{m=1}^{N} \bar{x}_{m} B_{m}(z),
$$

which shows that $f(z)$ is an algebraic function of the $A_{m}$ and $B_{m}$ $(m=1, \cdots, N)$.

4. Inequalities for the Faber polynomials. The extremum problems of the last paragraph were so easily solved since they led to a variational differential equation with a complete square expression. It will, therefore, be useful to discuss other extremum problems of the same class.

Consider, for example, the functional

$$
R(w, x)=\sum_{m, n=1}^{N} n c_{m n} x_{m} x_{n}+2 \sum_{m=1}^{N} x_{m} F_{m}[f(w)]-\log f^{\prime}(w)
$$

which depends upon the function $f(z)$, assumed to be of the class $\Phi$, and upon a fixed point $w \in D$. In view of (20), (21) and (23), we have for $R(w, x)$ the following variation formula: 


$$
R^{*}(w, x)=R(w, x)+a \rho^{2}\left(\sum_{m=1}^{N} x_{m} F_{m}^{\prime}\left(\zeta_{0}\right)+\frac{1}{f(w)-\zeta_{0}}\right)^{2}+o\left(\rho^{2}\right) .
$$

We see that $R(w, x)$ has a variation formula containing again a perfect square term and we expect, therefore, that the connected extremum problem will permit an easy solution. Therefore, we propose the following problem:

If $f(z)$ is an arbitrary function of the class $\Phi$, what is the maximum modulus of $R(w, x)$ and for which function $f(z)$ is this extremum attained?

Assuming that $f(z)$ is the desired extremum function and that it maps the original domain $D$ upon a domain $\Delta$, we find, by reiterating literally the conclusions of the last paragraph, that $\Delta$ is bounded by analytic slits $\zeta(s)$, each of which satisfies the differential equation

$$
R(w, x)^{-1} \cdot \zeta^{\prime}(s)^{2}\left[\sum_{m=1}^{N} x_{m} F_{m}^{\prime}[\zeta(s)]+\frac{1}{f(w)-\zeta(s)}\right]^{2}=1 .
$$

This equation may be integrated and yields

$$
\operatorname{Im}\left\{R(w, x)^{-1 / 2}\left[\sum_{m=1}^{N} x_{m} F_{m}(\zeta)-\log (\zeta-f(w))\right]\right\}=\text { const. }
$$

along each boundary continuum of $\Delta$. The function

$$
U(z)=R(w, x)^{-1 / 2}\left[\sum_{m=1}^{N} x_{m} F_{m}[f(z)]-\log (f(z)-f(w))\right]
$$

is regular throughout $D$, except for an $N$ th order pole at infinity and a logarithmic pole at $z=w$. It has a constant imaginary part along each boundary continuum of $D$.

In order to make use of this fact as in the last paragraph, we have to define another pair of functions. Let $A(z, w)$ and $B(z, w)$ be univalent functions of $z$ in $D$ which map it upon the whole $\zeta$-plane, slit along circular arcs with center at the origin, and along straight segments pointing to the origin respectively, and such that $w \in D$ corresponds to the origin in the $\zeta$-plane. We suppose at infinity the developments:

$$
\begin{aligned}
& \log A(z, w)=\log (z-w)+\sum_{n=1}^{\infty} \alpha_{n}(w) z^{-n} \\
& \log B(z, w)=\log (z-w)+\sum_{n=1}^{\infty} \beta_{n}(w) z^{-n} .
\end{aligned}
$$


On each boundary continuum of $D, \log A$ will have constant real parts while $\log B$ will possess constant imaginary parts. The functions

$$
\phi(z, w)=\frac{1}{2} \log [A(z, w) \cdot B(z, w)]=\log (z-w)+\sum_{n=1}^{\infty} a_{n}(w) z^{-n}
$$

and

$$
\psi(z, w)=\frac{1}{2} \log \frac{B(z, w)}{A(z, w)}=\sum_{n=1}^{\infty} b_{n}(w) z^{-n}
$$

are, therefore, regular in $D$ with the exception of the logarithmic poles at $w$ and $\infty$ for $\phi(z, w)$. On each boundary continuum of $D$, we have obviously:

$$
\phi(z, w)=\overline{\psi(z, w)}+\text { const. }
$$

where the constant depends on the particular boundary continuum and on $w$.

There exist, of course, close relations between the functions $\phi, \psi$ on the one hand, and the functions $A_{m}(z), B_{m}(z)$ on the other. In fact, let us consider the integral around the whole boundary $C$ of $D$ :

$$
\frac{1}{2 \pi i} \oint_{C} A_{m}(z) \phi^{\prime}(z, w) d z=A_{m}(w)+m a_{m}(w)-w^{m} ; \quad \phi^{\prime}=\frac{d}{d z} \phi
$$

The above equation is an elementary consequence of Cauchy's residue theorem. On the other hand, we have in view of (34), (50) and the single-valuedness in $D$ of all functions concerned:

$$
\begin{aligned}
\frac{1}{2 \pi i} \oint_{C} A_{m}(z) \phi^{\prime}(z, w) d z & =\frac{1}{2 \pi i} \oint_{c} \overline{B_{m}(z)} \overline{d \psi(z, w)} \\
& =-\frac{1}{2 \pi i} \oint_{c} B_{m} d \psi
\end{aligned}
$$

Again, we apply the residue theorem which shows that the above integral is zero, $B_{m}$ and $\psi$ being regular throughout $D$. Hence, we have proved:

$$
A_{m}(w)=-m a_{m}(w)+w^{m} .
$$

Using (33) and (49), we find, for fixed $w$ and $z$ near infinity, the development

$$
\phi(z, w)=\log (z-w)-\sum_{m, n=1}^{\infty} m^{-1} a_{m n} w^{-n} z^{-m} .
$$


Since $\left(n a_{m n}\right)$ is a symmetric matric, we see that $\phi(z, w)-\log (z-w)$ is a symmetric analytic function of $z$ and $w$.

Let us consider next the following consequence of the residue theorem:

$$
\frac{1}{2 \pi i} \oint_{C} A_{m}(z) \psi^{\prime}(z, w) d z=m b_{m}(w)
$$

In view of (34) and (50), we may write this integral also in the form:

$$
\begin{aligned}
\frac{1}{2 \pi i} \oint_{c} A_{m}(z) d \psi & =\frac{1}{2 \pi i} \oint_{c} \overline{B_{m}(z)} d \bar{\phi} \\
& =-\frac{1}{2 \pi i} \oint_{c} B_{m}(z) \phi^{\prime}(z, w) d z
\end{aligned}
$$

which gives, again because of the residue theorem,

$$
m b_{m}(w)=-\overline{B_{m}(w)} .
$$

Therefore, in view of $\left(33^{\prime}\right)$ and $\left(49^{\prime}\right)$ :

$$
\psi(z, w)=-\sum_{m, n=1}^{\infty} \frac{1}{m} b_{m n} w^{-n} z^{-m}=-\sum_{m, n=1}^{\infty} \frac{1}{m n} n b_{m n} \bar{w}^{-n} z^{-m} .
$$

Using the Hermitian character of $m b_{n m}$, we find finally:

$$
\psi(z, w)=-\sum_{m, n=1}^{\infty} \frac{1}{n} b_{n m} w^{-n} z^{-m} ;
$$

(57) and $\left(57^{\prime}\right)$ show that $\psi$ is analytic in $z$ and $w$.

Let us return now to the function $U(z)$, defined in (47). We prove easily, as in the last paragraph, the identity:

$$
\begin{array}{r}
R(w, x)^{-1 / 2}\left[\sum_{m=1}^{N} x_{m} F_{m}[f(z)]-\log (f(z)-f(w))\right] \\
=R(w, x)^{-1 / 2}\left[\sum_{m=1}^{N} x_{m} A_{m}(z)-\phi(z, w)\right] \\
\quad+\overline{R(w, x)^{-1 / 2}}\left[\sum_{m=1}^{N} \tilde{x}_{m} B_{m}(z)-\psi(z, w)\right] .
\end{array}
$$

Putting again

$$
e^{i r}=\operatorname{sgn} R(w, x),
$$

we may write $(58)$ in the form: 


$$
\begin{aligned}
\sum_{m=1}^{N} x_{m} F_{m} & {[f(z)]-\log (f(z)-f(w)) } \\
& =\sum_{m=1}^{N} x_{m} A_{m}(z)-\phi(z, w)+e^{i \tau}\left[\sum_{m=1}^{N} \bar{x}_{m} B_{m}(z)-\psi(z, w)\right] .
\end{aligned}
$$

Comparing the coefficients of $z^{-n}$ on both sides, we find in view of (2), (5), (33), $\left(33^{\prime}\right),(49),\left(49^{\prime}\right),\left(52^{\prime}\right)$ and (56):

$$
\begin{aligned}
\sum_{m=1}^{N} x_{m} c_{m n} & +\frac{1}{n} F_{n}[f(w)] \\
= & \sum_{m=1}^{N} x_{m} a_{m n}+\frac{1}{n} A_{n}(w)+e^{i r}\left[\left[\sum_{m=1}^{N} x_{m} b_{m n}+\frac{1}{n} \overline{B_{n}(w)}\right] .\right.
\end{aligned}
$$

Multiplying with $n x_{n}$ and summing up for $1 \leqq n \leqq N$, we get:

$$
\begin{aligned}
& \sum_{m, n=1}^{N} n c_{m n} x_{m} x_{n}+\sum_{m=1}^{N} x_{m} F_{m}[f(w)]=\sum_{m, n=1}^{N} n a_{m n} x_{m} x_{n} \\
& +\sum_{m=1}^{N} A_{m}(w) x_{m}+e^{i r}\left[\sum_{m, n=1}^{N} n b_{m n} \bar{x}_{m} x_{n}+\sum_{m=1}^{N} \overline{B_{m}(w)} x_{m}\right] .
\end{aligned}
$$

On the other hand, we obtain from (59) in the limit $z \rightarrow w$ :

$$
\begin{aligned}
\sum_{m=1}^{N} x_{m} F_{m}[f(w)]-\log f^{\prime}(w)= & \sum_{m=1}^{N} x_{m} A_{m}(w)-\chi(w) \\
& +e^{i \tau}\left[\sum_{m=1}^{N} \bar{x}_{m} B_{m}(w)-\psi(w, w)\right],
\end{aligned}
$$

where $\chi(w)=\lim _{z \rightarrow w}[\phi(z, w)-\log (z-w)]$. Adding together (61) and (62) yields:

$$
\begin{gathered}
\sum_{m, n=1}^{N} n c_{m n} x_{m} x_{n}+2 \sum_{m=1}^{N} x_{m} F_{m}[f(w)]-\log f^{\prime}(w) \\
(63)=\sum_{m, n=1}^{N} n a_{m n} x_{m} x_{n}+2 \sum_{m=1}^{N} x_{m} A_{m}(w)-\chi(w) \\
\quad+e^{i \tau}\left[\sum_{m, n=1}^{N} n b_{m n} \bar{x}_{m} x_{n}+2 \operatorname{Re}\left\{\sum_{m=1}^{N} \bar{x}_{m} B_{m}(w)\right\}-\psi(w, w)\right] .
\end{gathered}
$$

From (63) we derive: 


$$
\begin{aligned}
|R(w, x)| \leqq\left|\sum_{m, n=1}^{N} n a_{m n} x_{m} x_{n}+2 \sum_{m=1}^{N} x_{m} A_{m}(w)-\chi(w)\right| \\
+\mid \sum_{m, n=1}^{N} n b_{m n} \bar{x}_{m} x_{n} \\
+2 \operatorname{Re}\left\{\sum_{m=1}^{N} \bar{x}_{m} B_{m}(w)\right\}-\psi(w, w) \mid
\end{aligned}
$$

Since the right-hand side depends only on $D$ and not on the particular function $f(z)$, this inequality, proved for the maximum of $|R(w, x)|$ only, holds a fortiori for the rest of the family $\Phi$.

As before, we may improve our result and prove:

$$
\begin{aligned}
\mid R(w, x) & -\sum_{m, n=1}^{N} n a_{m n} x_{m} x_{n}-2 \sum_{m=1}^{N} x_{m} A_{m}(w)+\chi(w) \mid \\
& \leq\left|\sum_{m, n=1}^{N} n b_{m n} \tilde{x}_{m} x_{n}+2 \operatorname{Re}\left\{\sum_{m=1}^{N} \bar{x}_{m} B_{m}(w)\right\}-\psi(w, w)\right| .
\end{aligned}
$$

This result contains of course Grunsky's inequalities as particular cases. If, on the other hand, we put $x_{1}=x_{2}=\cdots=x_{N}=0$, we get from (65)

$$
\left|\log f^{\prime}(w)+\chi(w)\right| \leqq|\psi(w, w)|
$$

or, in view of (53) and $\left(57^{\prime}\right)$, the following inequality which is valid as long as all series concerned are convergent:

$$
\left|\log f^{\prime}(w)+\sum_{m, n=1}^{\infty} \frac{1}{m} a_{m n} w^{-(m+n)}\right| \leqq \sum_{m, n=1}^{\infty} \frac{1}{n} b_{n m} \bar{w}^{-n} w^{-m} .
$$

In the particular case that $D$ is the domain $|z|>1$, we have $a_{m n}=0$, $b_{m n}=\delta_{m n}$, whence:

$$
\left|\log f^{\prime}(w)\right| \leqq \log \frac{|w|^{2}}{|w|^{2}-1},
$$

a well known inequality.

It would be of interest to derive estimates for $F_{n}[f(w)]$ without combining this functional with other terms. But since the variational differential equation in this problem is much more complicated, no easy solution is to be expected. To indicate the difficulty of these questions, let us remark that the determination of exact bounds for $\left|F_{n}^{\prime}[f(w)]\right|$, even only in the case that $D$ is the exterior of the unit circle, would solve simultaneously the Bieberbach problem for func- 
tions which are univalent inside the unit circle. On the other hand, the determination of exact bounds for the coefficients $c_{m n}$ in the Faber development would lead to such bounds for the $c_{1 n}$, that is, the coefficients of univalent functions of the type (1). Since this problem is yet unsolved, we see that little progress is at the moment to be expected in the general question of the $c_{m n}$. It appears, therefore, particularly interesting that for the functionals discussed above such an easy and complete answer is possible. Numerous other functionals of such variational behavior might be constructed and new inequalities be established.

\section{BIBLIOGRAPHY}

1. H. Grunsky, Koeffizientenbedingungen fïr schlicht abbildende meromorphe Funktionen, Math. Zeit. vol. 45 (1939) pp. 29-61.

2. M. Schiffer, $A$ method of variation within the family of simple functions, Proc. London Math. Soc. (2) vol. 44 (1938) pp. 432-449.

3. - Variation of the Green function and theory of the p-valued functions, Amer. J. Math. vol. 65 (1943) pp. 341-360.

4. A. C. Schaeffer and D. C. Spencer, The coefficients of schlicht functions, Duke Math. J. vol. 10 (1943) pp. 611-635.

5. I. Schur, On Faber polynomials, Amer. J. Math. vol. 67 (1945) pp. 33-41.

HEBREW UNIVERSITY AND

HARVARD UNIVERSITY 